Proceedings of the 2012 Winter Simulation Conference

C. Laroque, J. Himmelspach, R. Pasupathy, O. Rose, and A. M. Uhrmacher, eds.

\title{
SIMULATION-BASED VALIDITY ANALYSIS OF ECOLOGICAL USER EQUILIBRIUM
}

\author{
Yun-Pang Flötteröd \\ Peter Wagner \\ Michael Behrisch \\ Daniel Krajzewicz \\ German Aerospace Center \\ Rutherfordstraße 2 \\ D-12489 Berlin, GERMANY
}

\begin{abstract}
Traffic is believed to organize itself by minimizing user's travel cost (travel time is used as cost very often). Almost all the analytical and numerical work in the last 50 years has concentrated on travel cost and the assumption that costs are always an increasing function of demand. With fuel consumption, this is no longer true, since there is typically an optimum speed where fuel consumption and emission production is minimized. This paper explores some of the implications when using fuel consumption as an objective function for achieving an user equilibrium traffic state. It turns out, that this leads to multiple solutions (multiple equilibriums) and sometimes counter-intuitive behavior, which makes it unlikely that such a scheme that minimizes fuel consumption alone will ever be implemented in real systems.
\end{abstract}

\section{INTRODUCTION}

Microscopic simulation technique has been extensively applied both in traffic modeling and traffic management to verify basic traffic phenomena, to explore and explain complex traffic phenomena which cannot be easily described by analytic models as well as to evaluate and optimize traffic management strategies. One of the main advantages to use microscopic simulation is that the interaction between the investigated moving objects, such as vehicles and pedestrians, and their fine moving behaviors, e.g., acceleration and deceleration, reaction times, safety gaps and lane-changing, can be accounted for and results in an overall system behavior close to reality. When analyzing traffic in networks, such an operative microscopic traffic model has to be enlarged by a strategic route choice model to describe how traffic is distributed in a network. The most often applied principle to model and optimize traffic is the user equilibrium (UE) principle based on Wardrop (1952). It states, that each of the users in such a network will minimize his or her travel time with the additional condition that no user can find a more favorable route to quickly reach his destination. Since the travel time is a monotonously increasing function of the demand for travel, it can be proved that at least for a constant demand a unique solution does exist and can be computed (Sheffi 1985).

However, travel-times are not the only objective to be minimized. In addition to travel times, the focus is now also moving towards reducing energy consumption and emissions. In many cities, traffic is managed more with the ecological concern, such as the low emission zones in Europe (European Union 2012). The objective is to maintain the sustainable living quality in cities by controlling the production of emissions. Generally, the evaluation of such ecological traffic management often adopts the aforementioned classical traffic simulation method and uses ecological measures, for instance fuel consumption and pollutants, instead of travel times. The monotonic character is however absent in the relationship between fuel consumption, traffic flows, traveling speed and acceleration. In most emission models, an ideal travel speed (around $60 \mathrm{~km} / \mathrm{h}$ for passenger cars) exists, speeds significantly smaller or larger increase fuel production and emissions. Therefore, it is doubtful if a unique and valid solution is still guaranteed with such direct 
Flötteröd, Wagner, Behrisch, and Krajzewicz

adaption and what problems under which circumstances will then arise. In the study from Behrisch, Flötteröd, Krajzewicz, and Wagner (2012), an analytical study with given link travel cost functions and a simplified speed-related fuel consumption equation has been put forward to check the validity of the classical UE principle in the ecological traffic management. The results showed that more than one UE solution is possible and some UE solutions could be unstable which depends on the used link travel cost function. At least the analytical method applied in their study does not include acceleration, therefore the current paper focus not only on the speed effect but also on the acceleration effect on the validity of ecological UE with the traffic simulation technique. The dynamic microscopic simulation tool SUMO (German Aerospace Center 2012) is used to examine the validity of ecological UE with a synthetic network.

\section{ANALYTICAL APPROACH}

Besides the weather- and pavement-related factors fuel consumption of a vehicle depends also on mechanical factors, such as vehicular type, brand and age, and on human factors, e.g., the choice of gear, acceleration strategies and so on. Therefore, it is not easy to comprehensively model vehicle fuel consumption in an analytical way. Following the approach of Treiber, Kesting, and Thiemann (2008) the fuel consumption of a vehicle principally depends on its current travel speed $v$ and acceleration $a$. When a vehicle travels with a constant speed $v$, the amount of fuel consumption per time unit can be calculated with the following equation

$$
e(v)=c_{0}+c_{1} v+c_{2} v^{2}+c_{3} v^{3}
$$

with an adequate parameters set of $\left\{c_{k}\right\}_{k=0,1,2,3}$. The total fuel consumption within a given time period $T$ is then

$$
E(T)=\int_{0}^{T} e(v(t))
$$

In the study from Behrisch, Flötteröd, Krajzewicz, and Wagner (2012) Equation (1) is simplified as $e(v)=c_{0}+c_{3} v^{3}$ so that the fuel consumption equation can be derived as a relatively function of traffic flows, shown in (3), with the given link travel time equation whose form is a function of traffic flows as well.

$$
e_{i}\left(q_{i}\right)=c T_{i}\left(1+k \frac{q_{i}}{q_{\infty}}+\frac{1}{2}\left(\frac{V_{i}}{v_{0}}\right)^{3} \frac{1}{\left(1+k \frac{q_{i}}{q_{\infty}}\right)^{2}}\right),
$$

where $e_{i}\left(q_{i}\right)$ is the fuel consumption on link $i$ with traffic flow $q_{i}, c$ is the fuel consumption when idling, $T_{i}$ is the travel time on link $i$ at free-flow speed, $k$ is the factor to show the relationship between travel time and traffic load (traffic flows), $V_{i}$ is the maximal travel speed, $v_{0}$ is the ideal travel speed and $q_{\infty}$ is the proxy of the capacity on link $i$. However, the fuel consumption function becomes more complicated, when a vehicle needs to accelerate. The respective fuel consumption can be calculated with the following general equation (Treiber, Kesting, and Thiemann 2008):

$$
e(v)=c_{0}+\left(c_{1}+c_{a v 1} a+c_{a v 2} a^{2}\right) v+c_{2} v^{2}+c_{3} v^{3},
$$

where $a$ is the acceleration and $c_{a v 1}$ and $c_{a v 2}$ are two additional parameters describing the relationship between acceleration and fuel consumption. It is clear to see that fuel consumption is proportional to acceleration. In this case, the deviation of an equation to obtain the form as the function of traffic flows is more complicated and leads to equations that are difficult to understand. Therefore, the simulation approach is adopted here. The calculation of fuel consumption is based on the HBEFA model (INFRAS 2012) which has already been implemented in SUMO. To capture the speed and acceleration effects on the 
Flötteröd, Wagner, Behrisch, and Krajzewicz

ecological UE two simulation experiments with typical traveling behaviors are set with use of a network of two one-lane routes with the same length $(10 \mathrm{~km})$.

\subsection{Speed-Only Effect}

In this experiment, the simulation network is designed such that the maximal travel speed on the two routes is $100 \mathrm{~km} / \mathrm{h}$. These two routes connect to a link with two lanes and a maximal travel speed of $30 \mathrm{~km} / \mathrm{h}$. The departure speed of each vehicle is set to the maximal travel speed. Therefore, only braking behavior with a very limited acceleration effect due to driving dynamic is expected in this network. With the use of the HBEFA model, the fuel consumption of each simulated vehicle is calculated mainly according to its vehicle type, vehicle age, travel speed and acceleration at each simulation step, i.e., 1 second, and collected on a link basis in each simulation run. Instead of using link travel time, the simulated fuel consumption on each link is used as link travel cost. Vehicles choose their routes in order to achieve less fuel consumption in their journeys. For analysis simplicity, only one passenger vehicle class is used in the experiment.

Furthermore, different traffic demands (traffic flows) are used to examine their effects on fuel consumption. The changes in fuel consumption with different shares on route 1 is first analyzed. To examine the speed effect on ecological UE, traffic assignments with different initial shares on route 1 are executed to find the ecological UE solutions. The logit model is used here for route choice and its mathematical form is shown in (5).

$$
p_{w}\left(r \mid C_{n, w}\right)=\frac{e^{-\theta_{w} V_{r n, w}}}{\sum_{j \in C_{n}} e^{-\theta_{w} V_{j n, w}}},
$$

where $p_{w}\left(r \mid C_{n, w}\right)$ is driver $w$ 's route choice probability to choose route alternative $r$ within his choice set $C_{n, w}, V_{r n, w}$ is the cost of the alternative $r$ in driver $w$ 's choice set $C_{n}$, i.e., the fuel consumption in our case, and $\theta_{w}$ is the factor to describe the driver $w$ 's cost perception. Here, all drivers have the same perception factor $\theta$ to keep the analysis simple. In the first simulation iteration, the vehicular route set with the given initial route share is assigned to the network. The resultant fuel consumption of each route can then be calculated according to the fuel consumed by the respective simulated vehicles. Based on the resultant fuel consumption on each route each driver updates his route choice probability $p_{w}\left(r \mid C_{n, w}\right)$, and a random number between 0 and 1 is generated to decide which route will be used in the next simulation iteration. For instance, if the route choice probabilities for route 1 and route 2 are 0.3 and 0.7 respectively and the generated random number is 0.2 , route 1 will be used. Such probability and route choice updating is iteratively executed during the simulation until the convergence is reached.

\subsection{Joint Effect of Speed and Acceleration}

Here, the network is almost the same one as that in experiment 1 except that the connecting link becomes a one-lane link with the maximal travel speed $100 \mathrm{~km} / \mathrm{h}$. Due to the reduction of the number of lanes on the connecting link a vehicle merging behavior appears in this network. Vehicles need to accelerate at the merge point, after they meet other vehicles on the other route. Such acceleration behavior results in an additional fuel consumption that is calculated with use of the HBEFA model in SUMO. The same analysis as that in the experiment "speed-only effect" is undertaken here as well.

\section{RESULTS OF THE COMPUTER EXPERIMENTS}

\subsection{Speed-Only Effect}

Figure 1 shows that vehicles consume much more fuel when the traffic demand is small. This is due to the fact that they travel almost with the maximal travel speed $(100 \mathrm{~km} / \mathrm{h})$. When the traffic demand reaches $1700 \mathrm{veh} / \mathrm{h}$, it shows that the minimal fuel consumption appears when only one route, either route 1 or 
route 2 , is used. An even share results in the maximal fuel consumption where the respective travel time is minimal and it is the classical UE solution regarding travel time. When the traffic demand increases to $3000 \mathrm{veh} / \mathrm{h}$, a plateau of less fuel consumption appears with a share between 0.45 and 0.55 on route 1 . Such a plateau area further expends when traffic demands are $4000 \mathrm{veh} / \mathrm{h}$ and $5000 \mathrm{veh} / \mathrm{h}$. It implies that different shares on route 1 results in the same fuel consumption and multiple UE solutions are possible. The initial route shares used in traffic assignment should therefore have an influence on searching the ecological UE solution.

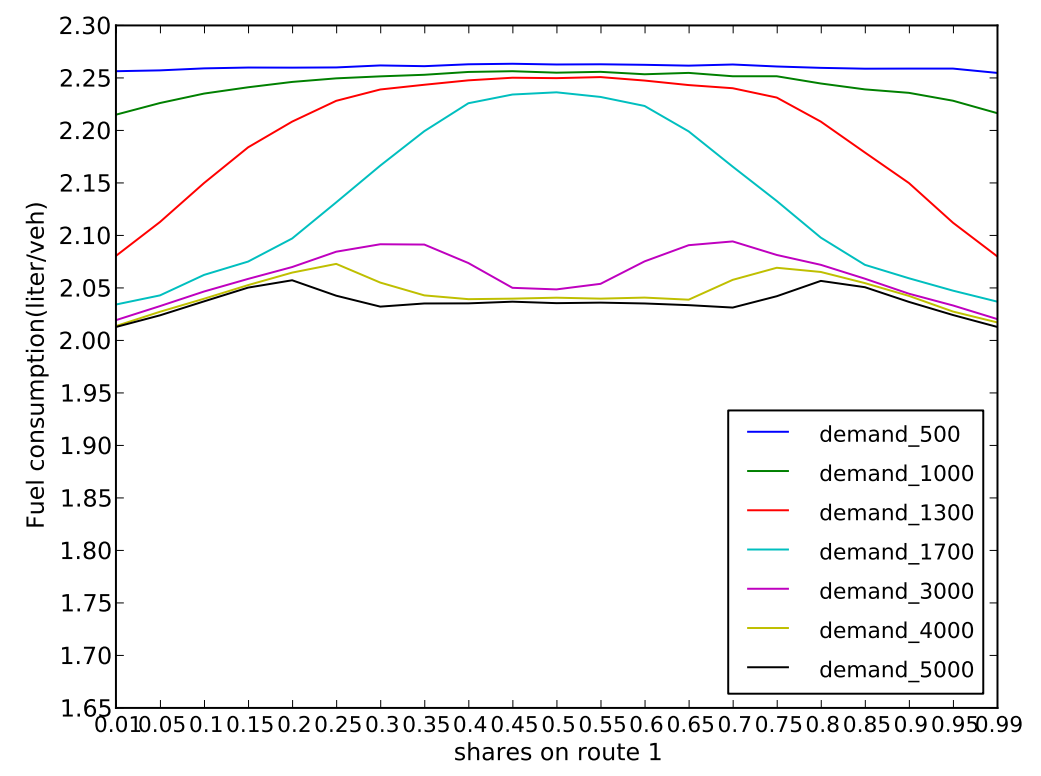

Figure 1: Simulated fuel consumptions with the speed-only effect.

Furthermore, traffic assignments with different initial route shares on route 1 are executed. The route choice results at the UE state in Figure 2 correspond to those found in Figure 1.

Given that two routes have the same length, the route choice on both route remains even with different initial route shares when the traffic demand is small, i.e., all vehicles travel with high speed (see Figure 2 (a)). When traffic demand further increases, the initial route shares begin to influence the final route choice. When traffic demand is $1700 \mathrm{veh} / \mathrm{h}$ and the initial share on route 1 is less than 0.4 , the UE solution is towards the use of route 2 and only less than $10 \%$ vehicles use route 1 . However, an initial share on route 1 greater than 0.5 results in a completely different route choice (see Figure 2 (b)). The influence of the initial route share becomes more significant with the traffic demand $5000 \mathrm{veh} / \mathrm{h}$. An even route share appears, when the initial route share is between 0.35 and 0.65 (see Figure 2 (c)). It is since there is a plateau region on the fuel consumption curve shown in Figure 1 and the search of a UE solution stays in this plateau region. Regarding the fuel consumption per vehicle at the UE state, more traffic demand results in lower travel speed and less fuel consumption (see Figure 3). The difference between the user equilibrium and the system optimum (SO), i.e., the total fuel consumption in the network is minimized, can also be observed in Figure 3. The ecological UE solution is to mainly use one route for achieving lower travel speed and the minimal fuel consumption, when traffic demand is less than $4000 \mathrm{veh} / \mathrm{h}$. In this case, drivers cannot find the other better route. Therefore, this ecological UE solution corresponds to the ecological system optimal (SO) solution. In comparison to that, the initial route shares between 0.35 and 0.65 with traffic demand $5000 \mathrm{veh} / \mathrm{h}$ results in an ecological UE solution that does not comply with the ecological SO solution and yields more fuel consumption than that with the other initial route shares. 


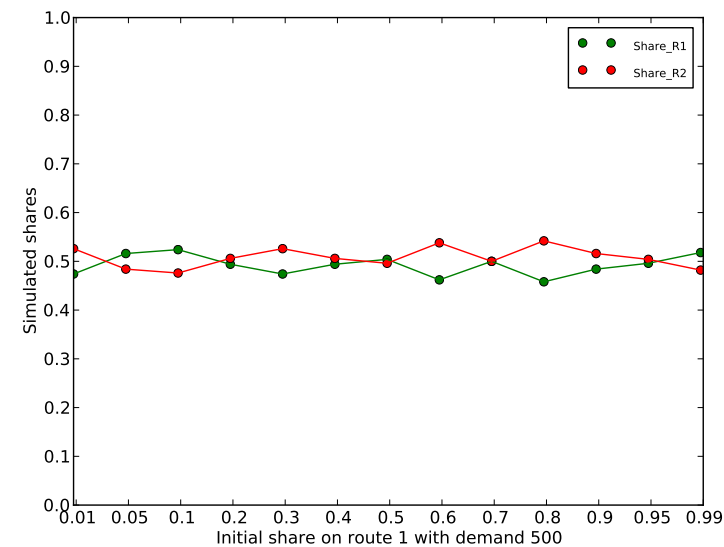

(a) Demand $=500 \mathrm{veh} / \mathrm{h}$

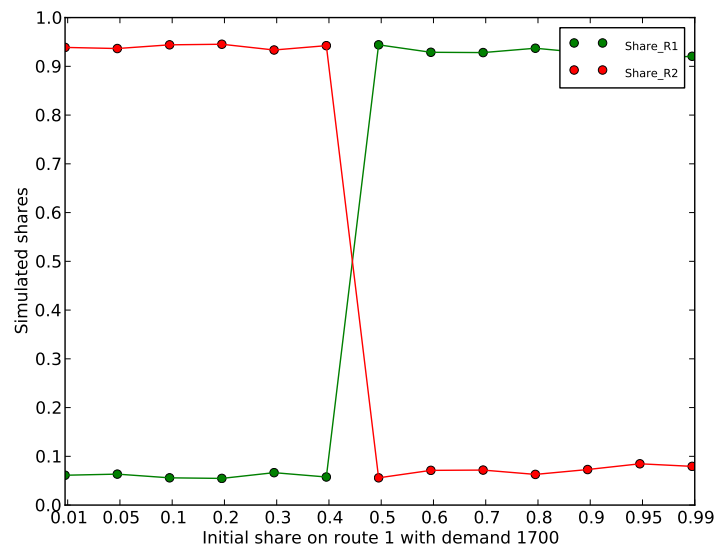

(b) Demand = $1700 \mathrm{veh} / \mathrm{h}$

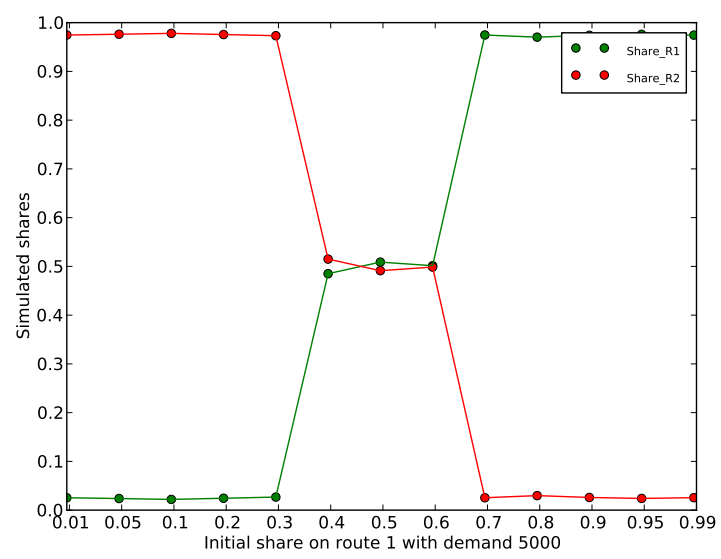

(c) Demand $=5000 \mathrm{veh} / \mathrm{h}$

Figure 2: Simulated route shares with the speed-only effect.

\subsection{Joint Effect of Speed and Acceleration}

Figure 4 shows the changes in fuel consumption with given route shares, when considering the speed and the acceleration effect. When only one route is mainly used, no vehicle merging appears and all vehicles travel with the maximal speed that results in a high fuel consumption. With large traffic demands vehicle merging becomes necessary and vehicles have to reduce their travel speeds and then accelerate to reach the desired speed, i.e., the maximal speed. Therefore, the minimal fuel consumption appears when the route share is even, i.e., vehicle merging frequently occurs. Moreover, there are some flat areas on the fuel consumption curves for the large traffic demands. These flat areas also imply that multi equilibriums are possible. In addition, in this simple network, the acceleration time is actually very short and its effect on fuel consumption is quite limited in comparison to the speed effect. Therefore, the above-mentioned speed-only effect can be observed again when traffic demand is not large, e.g., $1700 \mathrm{veh} / \mathrm{h}$. It is also indicated that less fuel consumption occurs with the route shares between 0.1 and 0.2 and between 0.8 and 0.9. The main reason is that the route capacity for free-flow speed in this network is around $900 \mathrm{veh} / \mathrm{h}$. With a even route share vehicles on both routes can travel almost with the maximal speed. Once the route share is considerable uneven, e.g., 85/15, vehicle merging behavior has more impact on vehicles on the 
Flötteröd, Wagner, Behrisch, and Krajzewicz

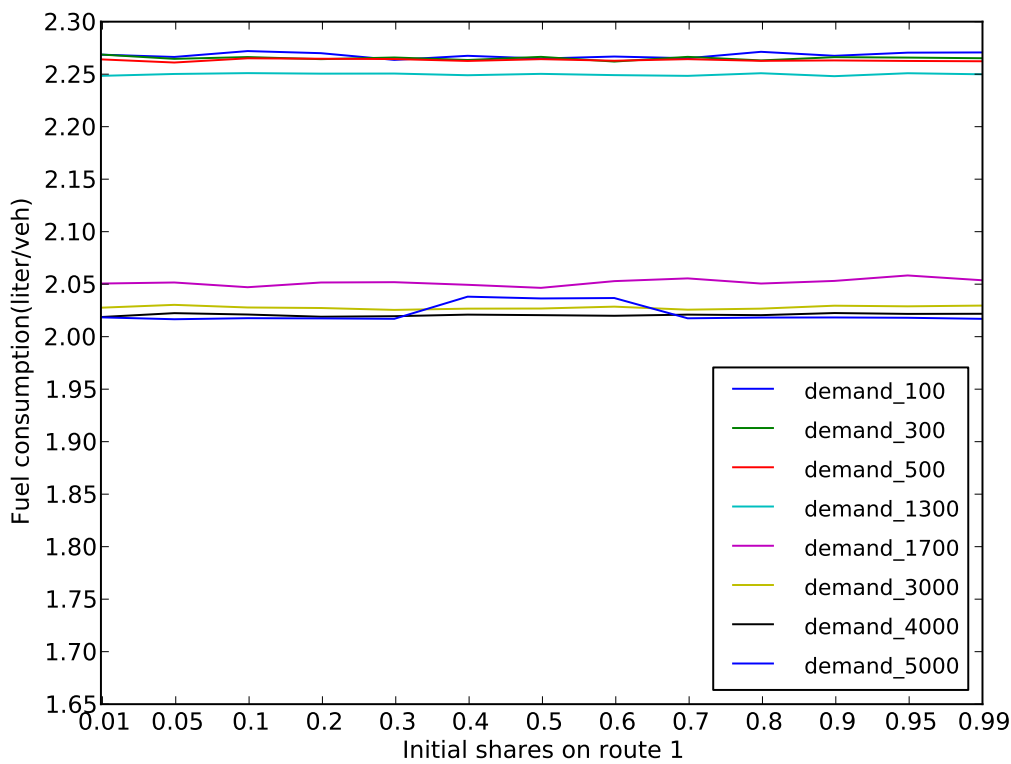

Figure 3: Fuel consumption at the UE state with the speed-only effect.

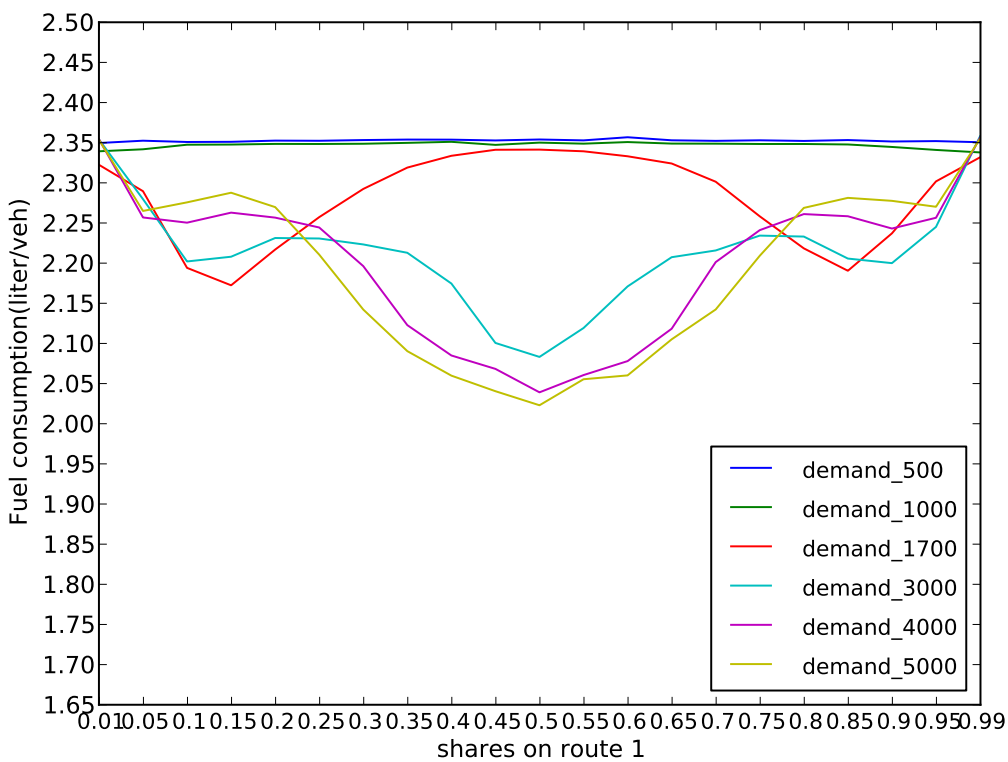

Figure 4: Simulated fuel consumptions with the vehicle merging effect. 
route with more traffic load such that they have to travel slowly and consume less fuel. The resultant simulated speed averages on the routes with different shares support this statement.

Furthermore, Figure 5 shows the traffic assignment results with different initial route shares. An even route share appears when traffic demand is $1000 \mathrm{veh} / \mathrm{h}$. Due to less traffic demand vehicle merging behavior rarely occurs and vehicles travel almost with the maximal speed. With traffic demand $1700 \mathrm{veh} / \mathrm{h}$ a even route share is obtained when the initial route share on route 1 is 0.01 or 0.99 . It is since these two initial route sets result in a network state that the travel speed and the fuel consumption on both routes are almost the same. The route choice probability on each routes is thus $50 \%$ which directly results in an UE state. However such a UE state results in a high fuel consumption (see Figure 6).

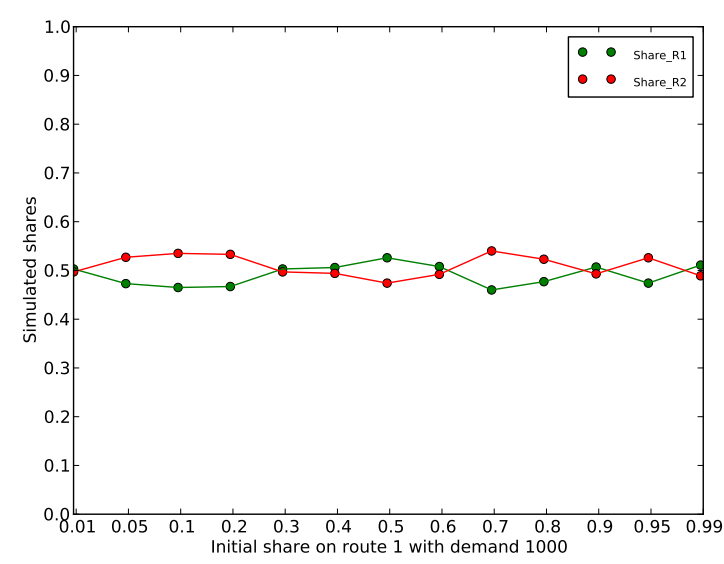

(a) Demand = $1000 \mathrm{veh} / \mathrm{h}$

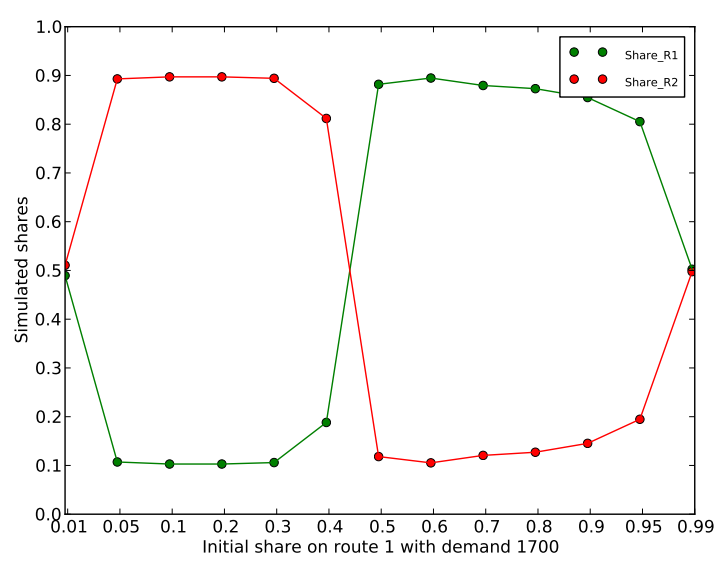

(b) Demand $=1700 \mathrm{veh} / \mathrm{h}$

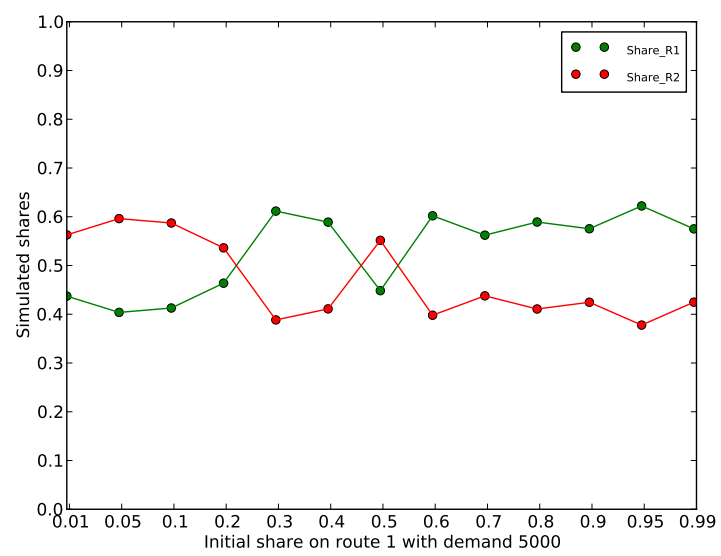

(c) Demand $=5000 \mathrm{veh} / \mathrm{h}$

Figure 5: Simulated route shares with the vehicle merging effect.

Regarding the other initial shares the ecological UE solution is found when one of the routes serves 80-90 percent of the traffic demand which corresponds to the results in Figure 4. When the traffic demand reaches $5000 \mathrm{veh} / \mathrm{h}$, a route share closed to a even share results in the ecological UE solution (see Figure 5 (d)) which complies with the classical UE solution. The resultant total fuel consumption per vehicle with different initial shares remains almost the same with slight fluctuation shown in Figure 6. 
Flötteröd, Wagner, Behrisch, and Krajzewicz

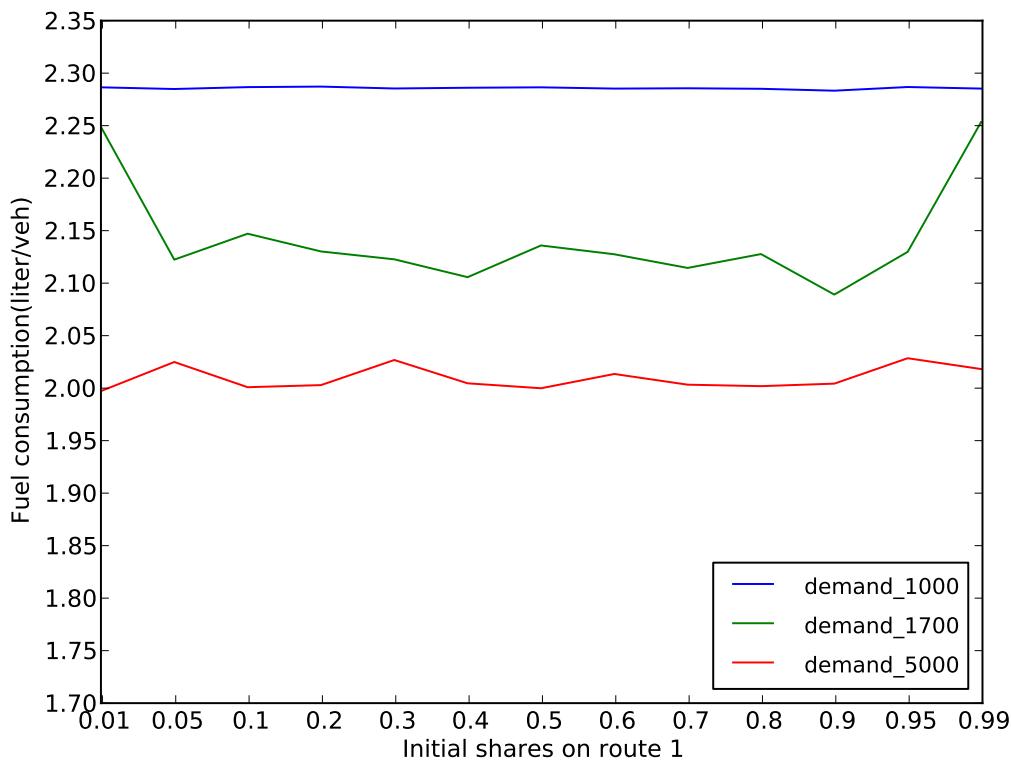

Figure 6: Fuel consumption at the UE state with the vehicle merging effect.

\section{CONCLUSIONS AND PERSPECTIVES}

The results from the simulation experiments show that a unique solution with use of Wardrop's UE principle for achieving a ecological user equilibrium is not guaranteed. Moreover, such an ecological UE solution is not stable and strongly depends on the given initial route sets, especially when only considering the speed effect. The results also demonstrate that by the use of fuel consumption as an objective function to compute a UE solution in networks makes the computation of such a UE much more complicated, since the monotonicity of the travel-time function is lost. It seems that this effect is also robust under the inclusion of acceleration. In our example, the captured acceleration effect is quite limited due to the network simplicity. Therefore, it is necessary to further investigate and verify the acceleration effect on the ecological user equilibrium with use of a real network. If the respective result would correspond to that with the speed-only effect, an ecological UE is difficult to be carried out in practice such that all vehicles on arterials, expressways or highways drive slowly in oder to couple the so-called ideal travel speed. Under this circumstance, an ecological UE could be realized only in a network with speed limits under the ideal travel speed, such as in a dense urban network. Otherwise, not only fuel consumption but also travel time need to be considered in the standard UE approach in favor of ecology.

\section{REFERENCES}

Behrisch, M., Y.-P. Flötteröd, D. Krajzewicz, and P. Wagner. 2012. "Ecological user equilibrium in traffic management?". Technical Report 76186, Institute of Transporation Systems, German Aerospace Center.

European Union 2012. "Low Emission Zones in Europe web site". Accessed Jun. 6, 2012. http://www. lowemissionzones.eu.

German Aerospace Center 2012. "SUMO: Simulation of Urban Mobility web site". Accessed Jul. 10, 2012. http://sumo.sourceforge.net.

INFRAS 2012. "HBEFA: Handbook Emission Factors for Road Transport web site". Accessed Jun. 24, 2012. http://www.hbefa.net/e/index.html. 
Sheffi, Y. 1985. Urban Transportation Networks: Equilibrium Analysis with Mathematical Programming Methods. Prentice-Hall.

Treiber, M., A. Kesting, and C. Thiemann. 2008, January. "How much does traffic congestion increase fuel consumption and emission? applying a fuel consumption model to the NGSIM trajectory data". In TRB 87th Annual Meeting Compendium of Papers CD-ROM, Number Paper No. 08-2715. Washington DC, USA.

Wardrop, J. G. 1952. "Some theoretical aspects of road traffic research". In Proceedings of the Institute of Civil Engineers, Volume 1. Delft, The Netherlands.

\section{AUTHOR BIOGRAPHIES}

YUN-PANG FLÖTTERÖD is researcher at the Institute of Transportation Systems at German Aerospace Center(DLR) since 2007. She received the doctoral degree from the Leibniz University Hanover, Germany, in 2007. She worked as a traffic planner for the THI traffic consulting company in Taiwan and was involved in different projects related to road planning, traffic planning and traffic safety. Her research interests include traffic demand modeling, traffic assignment modeling as well as the application of optimization methods in transportation. Her email address is yun-pang.floetteroed@dlr.de.

PETER WAGNER is Chief Engineer at DLR's Institute for Transportation Systems. He is working since 16 years now for DLR in varying positions ranging from a post-doctoral position to the management of a division. His main fields of competence are in the traffic modeling, traffic simulation and in traffic management, where he is author and co-author of many scientific papers. Before DLR, Dr. Wagner has worked in various post-doctoral positions with several German universities. Dr. Wagner earned his Ph.D. in 1990 from the University of Kiel in Germany. His email address is peter.wagner@ dlr.de.

MICHAEL BEHRISCH studied computer science at the Humboldt University Berlin and received his $\mathrm{PhD}$ on random graphs within the research center Matheon. Since 2007 he works at the German Aerospace Center where he is now team leader of the simulation group. His research interests are in algorithmic concepts for modeling and simulation, as well as driver models, routing and traffic assignment. He is also one of the main developers of the software package SUMO for microscopic traffic simulation. His email address is michael.behrisch@dlr.de.

DANIEL KRAJZEWICZ Dipl. Inf., Researcher at the German Aerospace Center; Born on 25.02.1972 in Bromberg, Poland. Finished the study of computer science with a Diploma degree in 2000. He works on the open source microscopic road traffic simulation package since 2001. Was involved in several traffic monitoring and management projects, mainly including OIS, INVENT, TrafficOnline, iTETRIS, and Pre-Drive C2X. Brings knowledge in traffic modeling, driver behavior, and large-scale traffic management. His email address is daniel.krajzewicz@dlr.de. 